\title{
TDT en España y el dividendo digital
}

\author{
Mercedes Román Portas \\ Universidad de Vigo \\ mroman@uvigo.es
}

Recibido: 26/07/2012

Aceptado: 11/10/2012

\begin{abstract}
Resumen
Este trabajo pretende conocer la evolución de la implantación de la Televisión Digital Terrestre en España. En este país conviven tres niveles territoriales: el estatal, el autonómico y el local. Cada uno con sus características, su legislación y su reparto de licencias. Este artículo se ciñe al ámbito estatal.

En la era digital se está prestando, más atención a la tecnología y a los soportes que a los contenidos. Aquí se parte de la idea de que los retos de la TDT se deberían centrar en mejorar la variedad y calidad de los contenidos y en potenciar la interactividad.

También se tratará la cuestión del dividendo digital. Antes del 2015 una banda de frecuencias debe quedar reservada para otros usos, en concreto para la prestación de servicios avanzados de comunicaciones electrónicas.
\end{abstract}

Palabras clave: Televisión digital terrestre, dividendo digital, interactividad

\section{DTT in Spain and the digital dividend}

\begin{abstract}
This paper aims to determine the evolution of the introduction of Digital Terrestrial Television in Spain. In this country, there are three territorial levels: state, regional, and local. Each of them with its own characteristics, legislation, and licensing division. This article refers to the state level.

In the digital age, more attention is being paid to technology and media than to contents. From here it's assumed that DTT challenges should focus on improving the variety and quality of content and promoting interactivity.

The issue of the digital dividend will also be addressed. Before 2015, a frequency band must be reserved for other uses, in particular for the provision of advanced electronic communications services.

Keywords: Digital terrestrial television, digital dividend, interactivity

\section{Referencia normalizada}

ROMÁN PORTAS, Mercedes (2012): “TDT en España y el dividendo digital”. Estudios sobre el mensaje periodístico. Vol. 18, núm. especial noviembre, págs.: 801-809. Madrid, Servicio de Publicaciones de la Universidad Complutense.
\end{abstract}

Sumario: 1. Introducción. 2. Metodología. 3. Aspectos legislativos relacionados con la TDT en España. 4. Distribución de canales digitales. 5. El reto de los contenidos y de la interactividad. 6. El dividendo digital. 7. Conclusiones. 8. Referencias bibliográficas

\section{Introducción}

Este trabajo, que es continuación de otros que he realizado y que se han publicado, pretende conocer la evolución de la implantación de la Televisión Digital Terrestre en España (Román, 2005 y 2008).

Los tres primeros países de la Unión Europea que afrontaron la implantación de la televisión digital terrestre (TDT) fueron el Reino Unido (1998), Suecia (1999) y España (2000). En el año 2001 lo harían Finlandia y Suiza, en el 2002 Alemania, en el 2003 Holanda y Bélgica, en 2004 Italia y en 2005 Francia y partir de entonces los demás. En España en el año 2000 nació la plataforma Quiero TV, de TDT con 14 canales de pago e interactivos. Esta plataforma, sin embargo, se cerró en el 2002. Hasta 
el año 2005 no se impulsó la TDT gratuita. Ese mismo año se estableció como fecha para el apagón analógico el 3 de abril del año 2010.

En España conviven tres niveles territoriales: el estatal, el autonómico y el local. Cada uno con sus características, su legislación y su reparto de licencias. Este artículo se ciñe al ámbito estatal.

En la era digital se está prestando, por el momento más atención a la tecnología y a los soportes que a los contenidos. En este artículo se parte de la idea de que los retos de la TDT se deberían centrar en mejorar la variedad y calidad de los contenidos y en potenciar la interactividad.

La distribución de contenidos en la actualidad es multisoporte, (teléfonos móviles, iPad, iphone, smartphone, etc.), multicanal (cable, satélite) y multiventanas. En este contexto la televisión digital sigue teniendo la centralidad en los hogares. Incluso para los mejor dotados y que cuentan con todo tipo de dispositivos, el televisor sigue siendo una plataforma que ahora además de TV proporciona Internet, redes sociales y servicios de valor añadido.

En mi opinión resultaron claves para la promoción de la TDT a los ciudadanos: que el apagón analógico tuviese fecha fija y fuese obligatorio; el desarrollo de la tecnología de televisión de pantallas planas; las ayudas autonómicas; el ajuste de precios de televisores y descodificadores y la planificación técnica y secuencial. Sin embargo parece que hubo cierta imprevisión económica y social, poco desarrollo de los servicios de valor añadido y como ya se ha señalado el no haber aprovechado hasta el momento las potencialidades de la interactividad.

\section{Metodología}

El método empleado en esta investigación ha sido el de búsqueda de datos e interpretación de los mismos. Se ha indagado en fuentes primarias, especialmente las legislativas que hacen referencia a la temática de estudio y en fuentes secundarias, libros y artículos publicados en revistas científicas en los últimos años.

\section{Aspectos legislativos relacionados con la TDT en España}

Tres años después del cierre de la plataforma Quiero TV, el 14 de junio de 2005, se aprobó la "Ley de medidas urgentes para el impulso de la Televisión Digital Terrestre"; que además liberalizaba la televisión por cable y afirmaba fomentar el pluralismo (Ley 10/2005, BOE 15 de junio de 2005). Esta ley se denominaba de "medidas urgentes" porque se adelantaba a Ley General del Audiovisual que de hecho no se aprobaría hasta el año 2010.

En el preámbulo de esta ley se hacía referencia a las dificultades habidas hasta entonces y a la necesidad de poner los medios para desarrollar la TDT.

"En el contexto actual, en el que la transición a la televisión y radio digital en España ha sufrido distintos avatares que vinieron a retrasar la implantación y despliegue de nuevos programas y servicios disponibles para todos los ciudadanos, se hace necesario articular medidas urgentes para favorecer la efectiva transición desde la tecnología analógica a la digital terrestre, ofrecer a los ciudadanos la posibilidad de acceder a un mayor número de programas y de servicios, con mayor calidad y garantizar la debida pluralidad de la oferta en España” (Ley 10/2005, BOE 15 de junio de 2005, pp. 20562 y ss) 
Esta ley eliminó la restricción de que hubiese sólo tres televisiones privadas. Un mes después de la publicación de la ley, el 29 de julio, se aprobó en un Real Decreto el "Plan técnico nacional de la televisión digital terrestre" (RD. 944/2005, BOE, 30 de julio de 2005, pp. 27006 y ss). Este Real Decreto dio lugar al relanzamiento de la TDT en España y adelantó el cese de las emisiones de la televisión analógica al 3 de abril de 2010 (la fecha límite en toda Europa era el 2012). Entre otras cosas estableció las condiciones del paso al sistema digital y al reparto de múltiplex a las cadenas de ámbito estatal.

"Cada una de las sociedades concesionarias, con arreglo a la Ley 10/1988, de 3 de mayo, de televisión privada, del servicio público de televisión con tecnología analógica de ámbito estatal existentes a la entrada en vigor de este real decreto accederá a un canal digital dentro de un múltiple digital de cobertura estatal, para permitir que emita simultáneamente con tecnología analógica y con tecnología digital" (BOE de 30 de julio de 2005, p. 27007)

Se legisló siguiendo un criterio técnico de la distribución total del espectro (Bustamante, 2009, 9) sin preocuparse tanto de su viabilidad económica:

"Cada una delas sociedades concesionarias del servicio público de televisión de ámbito estatal existentes a la entrada en vigor de este real decreto accederá, previa solicitud y tras el cese de las emisiones de televisión terrestre con tecnología analógica, a un múltiple digital de cobertura estatal" (Ibíd., p. 27008)

Quizá convenga dejar claras las definiciones de múltiple digital, canal digital y programa de televisión, tal como los describe la ley de 30 de julio de 2005.

Múltiple digital: señal compuesta para transmitir un canal o frecuencia radioeléctrica y que, al utilizar la tecnología digital, permite la incorporación de las señales correspondientes a varios canales de televisión y de las señales correspondientes a varios servicios asociados y a servicios de comunicaciones electrónicas.

Canal digital de televisión: parte de la capacidad de un múltiple digital que se utiliza para la incorporación en él de un programa de televisión.

Programa de televisión: organización secuencial en el tiempo de contenidos audiovisuales, puesta a disposición del público de forma independiente, bajo la responsabilidad de una misma persona y dotada de identidad e imagen propias.

Cada múltiple digital de cobertura estatal o autonómica integraría, inicialmente, al menos cuatro canales digitales susceptibles de ser explotados las 24 horas del día. Es decir, se sacó a concurso todo lo que cabía en el espectro y se dieron licencias de TDT a dos nuevas cadenas: Cuatro y La Sexta.

Este reparto generó poca rentabilidad a corto plazo y se produjeron fusiones como la de Cuatro y Telecinco. Se reservaron al Ente Público Radiotelevisión Española, para cuando se produjese el cese de las emisiones con tecnología analógica, dos múltiples digitales de cobertura estatal para su explotación en régimen de gestión directa. Además la televisión pública dejó de emitir publicidad, hecho que benefició a las empresas privadas.

Con un poco de perspectiva, en mi opinión, parece que quizá hubiese sido mejor haber repartido dos canales. De los cuatro canales adjudicados a cada operador, tres se subordinaban al generalista, siempre vinculado al entretenimiento, con un exceso 
de redifusiones en el resto de canales y con muchas limitaciones en la producción. Era, en definitiva, una televisión de poca diversidad y nada interactiva.

Aunque se buscaba más variedad, esta es una de las cosas que más se echan en falta. "En el escenario de transición de la televisión analógica a la televisión digital terrestre, se lleva a cabo la asignación de mayor capacidad de transmisión en la prestación de servicios de TDT para que, garantizando una mayor oferta televisiva, se difunda una programación novedosa, innovadora y diferenciada" (BOE de 30 de julio de 2005, p. 27006)

Con la TDT se prometía mayor calidad, interactividad, desarrollo de nuevos servicios, incrementar la oferta televisiva y el pluralismo, reforzando la libertad de elección de los ciudadanos en su acceso a los servicios de televisión. Se hablaba de que consolidaría un mercado de televisión más plural y más competitivo. Pero la TDT ahora mismo es más bien, un sistema ampliado del modelo anterior porque la excesiva preocupación por lo técnico y por cumplir los plazos se hizo a costa de las potencialidades de la interactividad y de la oferta y demanda de servicios avanzados. El servicio de TDT se explotaría en las siguientes bandas de frecuencias:

a) 470 a $758 \mathrm{MHz}$ (canales 21 a 56) se destinan al establecimiento de redes de televisión digital.

b) 758 a $830 \mathrm{MHz}$ (canales 57 a 65) se destinaron, principalmente, al establecimiento de redes de frecuencia única de ámbito territorial autonómico y provincial.

c) 830 a $862 \mathrm{MHz}$ (canales 66 a 69). Formaron cuatro múltiples digitales en redes de frecuencia única de ámbito estatal.

En el año 2007 se aprobó el Plan Nacional de transición a la TDT. En el año 2009 el Plan Avanza y el 31 de marzo de 2010 se aprobó la Ley General de Comunicación Audiovisual que acaba con la dispersión de leyes en este sector y de la que se quiere destacar lo siguiente:

Esta ley flexibilizó las medidas anti concentración de empresas privadas. Lo que favoreció las fusiones. También redujo el rol de RTVE y su doble financiación. Como consecuencia de ambas decisiones se reduce el pluralismo y el servicio público.

Por otra parte la ley preveía crear un consejo estatal de medios que fuera independiente (pero que hasta la fecha no se ha creado)

Igualmente promovía la producción y la edición propia de informativos (en la televisión pública)

Y eliminó el régimen de concesiones y obligó a obtener las licencias por concurso público.

Sin embargo, pocos meses después de la aprobación de la ley, en julio de 2010 el Gobierno hizo concesión sin concurso a 18 canales nacionales de televisión privada por 15 años. Esto entra en conflicto con el "dividendo digital". Actualmente, octubre de 2012 está pendiente de resolverse el recurso interpuesto por MassMedia Assistants ${ }^{1}$ ante el Tribunal Supremo.

${ }^{1} \mathrm{http}: / /$ www.massmediaassistants.com/news/82-el-desmadre-de-la-tdt.aspx (consultado el 16 de octubre de 2012). MassMedia Assistants es una compañía especializada en prestación de servicios jurídicos en materia de Telecomunicaciones y Nuevas Tecnologías 


\section{Distribución de canales digitales}

En el RD de 29 de julio de 2005 se acordó la asignación de mayor capacidad de transmisión en la prestación de servicios de TDT, para que garantizando una mayor oferta televisiva se diese un impulso decisivo al desarrollo e implantación de la TDT en España.

La tabla 1 muestra el reparto realizado en el año 2005 de canales digitales a los operadores de televisión y su ubicación en los múltiples. Esta concesión fue otorgada por un período de 10 años.

Tabla 1: Concesión de canales digitales en el año 2005

\begin{tabular}{|l|l|l|l|}
\hline Red de cobertura estatal & Canal 67 & Canal 68 & Canal 69 \\
\hline RTVE & Sogecuatro & Telecinco & Antena 3 \\
RTVE & Sogecuatro & Telecinco & Antena 3 \\
RTVE & Sogecuatro & Telecinco & Antena 3 \\
RTVE & La Sexta & NET TV & VEO TV \\
& La Sexta & NET TV & VEO TV \\
& La Sexta & & \\
\hline
\end{tabular}

Fuente: elaboración propia a partir del RD 944/2005, BOE de 30 de julio de 2005

Este RD preveía que tras el cese de las emisiones analógicas, cada sociedad concesionaria de servicio público de televisión terrestre de ámbito estatal accedería, previa solicitud, a un múltiple digital de cobertura estatal. También establecía que la Corporación de Radio Televisión Española podría acceder a dos múltiples digitales de cobertura estatal para su explotación en régimen de gestión directa.

En el año 2010 se amplió el reparto anterior y el tiempo de la concesión que se hizo por 15 años (tabla 2). Con esta distribución se podría objetar que se cierra el mercado a otros operadores.

Tabla 2: Concesión de canales digitales en el año 2010

\begin{tabular}{|l|l|l|l|}
\hline RGE2 & $\begin{array}{l}\text { MPE1 } \\
\text { Sogecable, S.A. }\end{array}$ & $\begin{array}{l}\text { MPE2 } \\
\text { Gestevisión Telecinco, S.A. }\end{array}$ & $\begin{array}{l}\text { MPE3 } \\
\text { Antena 3 de Televisión, S.A. }\end{array}$ \\
\hline RTVE & Sogecuatro & Telecinco & Antena 3 \\
RTVE & Sogecuatro & Telecinco & Antena 3 \\
& La Sexta & NET TV & VEO TV \\
& La Sexta & NET TV & VEO TV \\
& La Sexta & & \\
\hline
\end{tabular}

Fuente: elaboración propia a partir del Real Decreto 365/2010, de 26 de marzo (BOE de 3 de abril de 2010)

Es posible que se modifique el reparto de canales como consecuencia del recurso antes mencionado. El Tribunal Supremo estudia la decisión del Gobierno socialista de otorgar 17 canales digitales a los concesionarios (Antena 3, Tele 5 (Mediaset España), Cuatro, la Sexta, Vocento y Unedisa) sin realizar el concurso público.

De prosperar el recurso, todos los concesionarios volverían a la situación de 2005 y el Gobierno se vería forzado a organizar el correspondiente concurso para la asig- 
nación de, al menos, 17 canales digitales. Existe pues una posibilidad de deshacer la lesiva política audiovisual de los últimos años.

\section{El reto de los contenidos y de la interactividad}

La TDT implica cambios en el modelo de entender y producir contenidos para televisión, puesto que integra servicios de valor añadido, el más relevante la interactividad (García, 2012).

El verdadero eje de la digitalización de la televisión lo constituyen los contenidos; sin embargo estos han sido los grandes olvidados en las políticas de comunicación sobre la TDT en España (Marzal y Casero: 2009, 102). Se ha constatado que el hecho de tener un mayor número de canales no produce necesariamente una mayor diversidad; a veces se ha conseguido incluso lo contrario.

La faltad de interactividad se debe entre otras cosas al tipo de descodificadores que las personas adquirieron. Para poder acceder a aplicaciones interactivas harían falta descodificadores MHP (Multimedia Home Platform) que en España apenas se vendieron. En Italia, por ejemplo, el gobierno de Berlusconi subvencionó este tipo de descodificadores y por eso están mucho más extendidos que en España. (Fernández, et al.: 2009, 36).

Otro problema que afecta a la interactividad es que apenas se producen programas interactivos. Un ejemplo de este tipo de programa fue el Sports ITV, que ofreció deporte interactivo durante las Olimpiadas en Pekín (Vivar y García: 2009, 207 y ss.), pero fue un caso aislado.

\section{El dividendo digital}

La implantación de la TDT afronta el problema del llamado dividendo digital. Un RD (BOE del 3 de abril de 2010) estableció que antes del 1 de enero de 2015 la sub banda de frecuencias (790-862 MHz) debía quedar reservada para otros usos y servicios, en concreto para la prestación de servicios avanzados de comunicaciones electrónicas ${ }^{2}$.

Por tanto, las cadenas de televisión tienen que abandonar antes del 1 de enero de 2015 las amplias frecuencias que ocupan a favor de las empresas de telecomunica-

${ }^{2}$ Con el objetivo de que la sub banda de frecuencias de 790 a $862 \mathrm{MHz}$ (canales radioeléctri$\cos 61$ a 69) pueda quedar reservada antes del 1 de enero de 2015, para otros usos y servicios se llevarán a cabo las siguientes actuaciones: Se planificarán tres nuevos múltiples digitales de cobertura estatal dirigidos a sustituir a los múltiples digitales asociados a los canales radioeléctricos 67, 68 y 69.La planificación de estos tres nuevos múltiples digitales se realizará por la Agencia Estatal de Radiocomunicaciones de acuerdo con los siguientes criterios:

a. Se establecerá una red de ámbito estatal tomando como base las áreas geográficas definidas por los proyectos técnicos identificados en el Plan Nacional de Transición a la Televisión Digital Terrestre, aprobado por Acuerdo del Consejo de Ministros de 7 de septiembre de 2007.

b. En cada una de estas áreas geográficas se establecerá una red de frecuencia única.

c. En la planificación de este nuevo múltiple digital no se utilizarán canales radioeléctricos de la sub banda de frecuencias de 790 a $862 \mathrm{MHz}$ (canales radioeléctricos 61 a 69). 
ciones. Este es un proceso previsto por la Agenda Digital Europea y por la Ley de Economía Sostenible aprobado por el anterior gobierno socialista.

En esta regulación y planificación radioeléctrica de los múltiples digitales, se están teniendo en cuenta las decisiones regulatorias de los organismos internacionales especializados en telecomunicaciones y las instituciones comunitarias. Estas instituciones han establecido que las frecuencias de 790 a $862 \mathrm{MHz}$ (que se corresponden con los canales radioeléctricos 61 a 69), puedan ser utilizadas para comunicaciones electrónicas de carácter paneuropeo. Esa banda de frecuencias es lo que se conoce como Dividendo Digital.

Los canales afectados por el dividendo digital son en su mayoría canales privados de ámbito nacional, aunque, en algunas regiones podría afectar a canales de TVE y a canales locales (RD 365/2010, BOE de 3 de abril de 2010).

Un acuerdo del Consejo de Ministros de 18 de noviembre de 2011 aprobó el Plan Marco de Actuaciones para la Liberación del Dividendo Digital. Este Plan Marco establece la hoja de ruta que permitirá liberar la banda de $800 \mathrm{MHz}$ para la prestación de servicios de comunicaciones electrónicas, en especial, la banda ancha ultrarrápida en movilidad. El objetivo es que cada ciudadano europeo tenga un ancho de banda de 30 megas por segundo en 2020 .

El gobierno actual tanteó a las televisiones privadas para que devolviesen la mitad de sus canales de TDT antes de 2015 con el fin de ahorrar costes en la reordenación del espectro radioeléctrico. Pero como estas empresas se resistieron, se buscó otra alternativa para evitar un enfrentamiento del Gobierno con las empresas de televisión ${ }^{3}$.

En septiembre de 2011 hubo una subasta entre las empresas de telecomunicación para la concesión de las bandas de $800 \mathrm{mhz}, 900 \mathrm{mhz}$ y 2,6 ghz (Orden ITC/2508/2011, de 15 de septiembre; BOE de 22 de septiembre)

Fueron admitidos a participar en la subasta los siguientes licitadores:

- «Vodafone España, S. A. U.»

- «Telefónica Móviles España, S. A. U.»

- «Euskaltel, S. A.»

- «France Telecom España, S. A. U.»

- «Telecom Castilla-La Mancha, S. A.»

- «Cableuropa, S. A. U.» (ONO).

- «Jazz Telecom, S. A. U.»

- «R Cable Telecomunicaciones Galicia, S. A.»

- «Consorcio de Telecomunicaciones Avanzadas, S. A.» (COTA).

- «TeleCable de Asturias, S. A. U.»

- «Obras Públicas y Telecomunicaciones de Navarra, S. A.» (OPNATEL).

Hay que tener en cuenta que la actividad de difusión de la TDT representa un porcentaje alto del negocio de telecomunicaciones de la compañía Abertis, alrededor del $40 \%$. Las televisiones abonan cinco millones de euros por canal en concepto de licencia.

${ }^{3}$ http://www.elconfidencial.com. 29 de marzo de 2012 
En abril del 2012 la secretaría de Estado de Telecomunicaciones y de la Sociedad de la Información confiaba en llegar a un acuerdo con las cadenas de televisión y el sector audiovisual para poder configurar un nuevo mapa digital español y poder adelantar en un año, la llegada de la telefonía de cuarta generación a España. Para poder implantar esta telefonía las cadenas actuales deberán trasladarse a otras frecuencias para poder destinar las que ahora utilizan a la transmisión de datos.

"La transición de los canales a otro lugar del espectro costará al Gobierno alrededor de 800 millones de euros, razón por la cual este busca alternativas que le permitan abaratar ese coste. La principal propuesta realizada al sector audiovisual es la de eliminar algunos de los canales de TDT existentes en la actualidad, lo que le permitiría reducir el importe del cambio. Fuentes del sector audiovisual aseguran que las cadenas están estudiando distintos planteamientos para brindarle al Ejecutivo alternativas sin necesidad de que ninguna cadena tenga que renunciar a los canales que tiene operativos" (La Voz de Galicia, 25 de abril de 2012).

Finalmente se llegó a un acuerdo (Consejo de ministros del 24 de agosto de 2012) aceptable para las televisiones privadas ${ }^{4}$.

\section{Conclusiones}

Del estudio realizado se han podido extraer las siguientes conclusiones:

En España se han adjudicado todos los canales digitales a un número reducido de operadores. Como consecuencia pocos actores controlan un gran número de canales.

En la actualidad se puede afirmar que la proliferación de canales no trajo consigo mayor variedad ni mejor calidad de contenidos.

El panorama español ha quedado configurado con más prestadores de servicios que titulares de empresas, ya que se han realizado algunas fusiones.

Se ha dejado de lado el desarrollo del potencial interactivo de la TDT y generalmente se siguen consumiendo los mismos contenidos.

En este contexto predomina la visión económica y tecnológica sobre la preocupación por una información de calidad, la protección a la diversidad, etc.

Se ha detectado un gran respeto a los intereses de los grandes grupos de comunicación. Los gobiernos han evitado la confrontación directa con ellos.

Los grandes grupos empresariales de comunicación reciben un gran porcentaje de la facturación por publicidad especialmente desde que no se emite publicidad en la televisión pública.

En suma: Los contenidos han sido los grandes olvidados, no se han potenciado los servicios interactivos y un reto pendiente es la TDT móvil.

\section{Referencias bibliográficas}

BUSTAMANTE RAMÍREZ, Enrique (2009): "La investigación en España sobre TDT“. Sphera Pública, no 9. Murcia, Servicio de publicaciones de la UCAM.

${ }^{4}$ http://www.cincodias.com, 25 de agosto de 2012 (consultado el 17 de octubre de 2012) 
GARCÍA GONZÁLEZ, Aurora (2012): En torno a la radio. Saarbrücken, Alemania, EAE.

FERNÁNDEZ ALONSO, Isabel et al. (2009): "Políticas de implantación de la TDT en Europa y efectos sobre la reconfiguración de los mercados de televisión. Los casos de Reino Unido, Francia, Italia y Alemania“, en Sphera Publica, n 9. Murcia, Servicio de Publicaciones de la UCAM.

MARZAL FELICI, Javier. y CASERO RIPOLLÉS, Andreu (2009): "Las políticas de comunicación ante la implantación de la TDT en España. Balance crítico y retos pendientes“, en Sphera Pública, no 9. Murcia, Servicio de publicaciones de la UCAM.

ROMÁN PORTAS, Mercedes (2005): "La televisión local en España“, en Sphera Publica $\mathrm{n}^{\mathrm{0}}$ 5. Murcia, Servicio de Publicaciones de la UCAM.

ROMÁN PORTAS, Mercedes (2008): "Televisión digital terrestre local en Galicia“, en Ámbitos. Revista Internacional de Comunicación, $\mathrm{n}^{\circ}$ 17. Sevilla, Universidad de Sevilla.

VIVAR ZURITA, Hipólito y GARCÍA GARCÍA, Alberto (2009): "La interactividad: concepto y factor de impulso de la TDT. Un caso práctico: Proyecto Sports ITV“, en Sphera Publica, $\mathrm{n}^{\circ}$ 9. Murcia, Servicio de Publicaciones de la UCAM.

\section{$\overline{\text { Mercedes ROMÁN PORTAS }}$}

mroman@uvigo.es

Facultad de Ciencias Sociales y de la Comunicación. A Xunqueira s/n. 36005 Pontevedra Universidad de Vigo

Profesora titular del área de periodismo 